\title{
Dickkopf1 Up-Regulation Induced by a High Concentration of Dexamethasone Promotes Rat Tendon Stem Cells to Differentiate Into Adipocytes
}

\author{
Wan Chen ${ }^{a}$ Hong Tang ${ }^{a}$ Xiangzhou Liua Mei Zhou ${ }^{a} \quad$ Jiqiang Zhang ${ }^{b}$ \\ Kanglai Tang ${ }^{a}$ \\ aDepartment of Orthopedic Surgery, Southwest Hospital, Third Military Medical University, Chongqing, \\ ${ }^{b}$ Department of Neurology, Third Military Medical University, Chongqing, China
}

\section{Key Words}

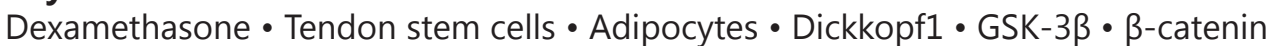

\begin{abstract}
Background/Aims: Dexamethasone (Dex)-induced spontaneous tendon rupture and decreased self-repair capability is very common in clinical practice. The metaplasia of adipose tissue in the ruptured tendon indicates that Dex may induce tendon stem cells (TSCs) to differentiate into adipocytes, but the mechanism remains unclear. In the present study, we used in vitro methods to investigate the effects of Dex on rat TSC differentiation and the molecular mechanisms underlying this process. Methods: First, we used qPCR and Western blotting to detect the expression of the adipogenic differentiation markers aP2 and C/EBP $\alpha$ after treating the TSCs with Dex. Oil red staining was used to confirm that high concentration Dex promoted adipogenic differentiation of rat TSCs. Next, we used qPCR and Western blotting to detect the effect of a high concentration of dexamethasone on molecules related to the canonical WNT/ $\beta$-catenin pathway in TSCs. Results: Treating rat TSCs with Dex promoted the synthesis of the inhibitory molecule dickkopf1 (DKK1) at the mRNA and protein levels. Western blotting results further showed that Dex downregulated the cellular signaling molecule phosphorylated glycogen synthase kinase-3 $\beta$ (P-GSK-3 $\beta$ (ser9)), upregulated P-GSK$3 \beta$ (tyr216), and downregulated the pivotal signaling molecule $\beta$-catenin. Furthermore, DKK1 knockdown attenuated Dex-induced inhibition of the canonical WNT/ $\beta$-catenin pathway and of the adipogenic differentiation of TSCs. Lithium chloride ( $\mathrm{LiCl}$, a GSK-3 $\beta$ inhibitor) reduced Dexinduced inhibition of the classical WNT/ $\beta$-catenin pathway in TSCs and of the differentiation of TSCs to adipocytes. Conclusion: In conclusion, by upregulating DKK1 expression, reducing the level of P-GSK-3 $\beta$ (ser9), and increasing the level of P-GSK-3 $\beta$ (tyr216), Dex causes the degradation of $\beta$-catenin, the central molecule of the classical WNT pathway, thereby inducing rat TSCs to differentiate into adipocytes.
\end{abstract}

Dr. Tang and Dr. Zhang

KARGER 125
Department of Orthopedic Surgery, Southwest Hospital, Third Military Medical University, Gaotanyan Street. 30, Shapingba District, Chongqing 400038, (China); or Department of Neurobiology, Third Military Medical University, Chongqing 400038, (China)

E-Mail tangkanglai@hotmail.com and zhangjgtmmu@yahoo.com 


\section{Cellular Physiology Cell Physiol Biochem 2015;37:1738-1749

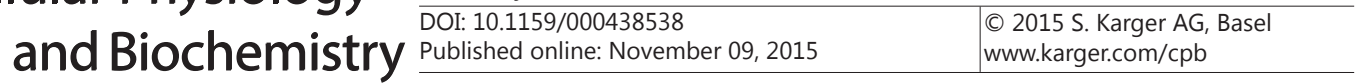 \\ Chen et al.: Dickkopf1 Promotes rTSCs to Differentiate into Adipocytes}

\section{Introduction}

Due to its anti-inflammatory and immunosuppressive effects, glucocorticoid has been widely used in the treatment of tendon disease and autoimmune diseases. Local injection of glucocorticoid is effective for reducing inflammation and improving pain symptoms in the treatment of early tendon disease. Long-term local injection of glucocorticoid to treat tendon disease or systemic use to treat tendon rupture caused by autoimmune disease is very common in clinical practice [1-3]. Clinical investigation has shown that glucocorticoidinduced tendon rupture is accompanied by adipose metaplasia $[4,5]$. Compared with tendon rupture caused by trauma, glucocorticoid-induced tendon rupture has a poor surgical repair rate, and it is prone to repeated rupture over the long term, indicating that glucocorticoid reduces the self-repair capability of the tendon. The irreplaceability of glucocorticoid in clinical practice and the challenge of the clinical treatment of glucocorticoid-induced tendon rupture make it necessary to investigate the mechanisms underlying glucocorticoid-induced tendon rupture, the adipose metaplasia, and the reduced self-repair capability so that an intervention strategy targeting the side effects of glucocorticoid on tendon can be identified.

It is well known that the maintenance and repair of adult tissues relies on small populations of resident stem cells [6, 7]. Recently, a group of adult stem cells has been found in human, mouse [8] and rat tendon [9]; this group of cells was therefore named tendon stem cells (TSCs). Tendons are maintained in a dynamic balance between microdamage and self-repair [10]. TSCs have a strong ability to proliferate and to differentiate into tendon cells, and such differentiation is very important for the self-renewal and repair of tendon $[11,12]$. However, under abnormal conditions, TSCs can also differentiate into cells of other mesodermal tissues, such as bone, cartilage, and adipocytes [8,9]. When TSCs differentiate into non-tendon cells, the self-repair ability of tendon is reduced $[11,13]$. Our previous studies found that Dex inhibits TSC differentiation into tendon cells by down regulating scleraxis. Zhang found that Dex treatment of human TSCs increases the levels of PPAR $\gamma$, a marker of adipogenic differentiation. When Dex-treated TSCs were implanted subcutaneously into rats, adipose tissue formation was observed through oil red staining [13]. The Dex-induced inhibition of TSC differentiation into tendon cells and promotion of differentiation into adipocytes may be important means by which Dex causes spontaneous tendon rupture and impairs the self-repair function. However, the underlying molecular mechanism has not been reported.

The differentiation of stem cells into adipocytes is mainly regulated by BMPs or the classical WNT signaling pathway [14]. The Wnt/ $\beta$-catenin signaling pathway plays an important role in regulating adipogenesis. Under normal culture conditions, the WNT signaling pathway and adipogenic pathway are reciprocally regulated [15]. Factors that inactivate the Wnt/ $\beta$-catenin pathway, including DKK1, are known to promote adipogenesis in vitro. In the present study, we demonstrate that by promoting DKK1 expression, reducing the level of P-GSK-3 $\beta$ (ser9), and increasing the level of P-GSK-3 $\beta$ (tyr216), Dex causes the degradation of $\beta$-catenin, the central molecule of the canonical WNT pathway, thereby promoting TSCs to differentiate into adipocytes.

\section{Materials and Methods}

Isolation and culture of rat TSCS

All experiments were approved by the Animal Research Ethics Committee of Third Military Medical University, China. Rat TSCs were isolated from Sprague-Dawley rats and cultured as described previously [16]. Briefly, the whole, intact flexor tendon was excised from both limbs of each rat following euthanasia. Only the midsubstance tissue was collected, and peritendinous connective tissue was carefully removed. The tissues were minced in sterile phosphate-buffered saline (PBS), digested for $2.5 \mathrm{~h}$ at $37^{\circ} \mathrm{C}$ with type I collagenase ( $3 \mathrm{mg} / \mathrm{ml}$ Sigma-Aldrich, St. Louis, MO, USA) and then passed through a 70-mm cell strainer (Becton Dickinson, Franklin Lakes, NJ, USA) to yield a single-cell suspension. The released cells were washed 


\section{Cellular Physiology Cell Physiol Biochem 2015;37:1738-1749 \begin{tabular}{l|l|l} 
DOI: 10.1159/000438538 & (C) 2015 S. Karger AG, Basel
\end{tabular} and Biochemistry Published online: November 09, $2015 \quad$ www.karger.com/cpb \\ Chen et al.: Dickkopf1 Promotes rTSCs to Differentiate into Adipocytes}

in PBS followed by centrifugation at $300 \mathrm{~g}$ for $5 \mathrm{~min}$ and then resuspended in Dulbecco's modified Eagle's medium (Gibco, Carlsbad, CA, USA) containing 10\% fetal bovine serum, $100 \mathrm{U} / \mathrm{ml}$ penicillin, $100 \mathrm{mg} / \mathrm{ml}$ streptomycin and $2 \mathrm{mM}$ L-glutamine (all from Invitrogen, Carlsbad, CA, USA). The isolated cells were diluted to different cell densities and cultured at $37^{\circ} \mathrm{C}$ in $5 \% \mathrm{CO}_{2}$ to form colonies. At day 2 after initial plating, the cells were washed twice with PBS to remove nonadherent cells. At day 7, the cells were trypsinized and mixed together as passage 0 (P0) cells [16]. Cells from passage 3 (P3) were used for subsequent experiments. TSCs were seeded onto 6-well plates for mRNA extraction, 10-cm-diameter petri dishes for protein extraction and coverslips in 24-well plates for oil red staining and immunostaining. Culture medium with or without $1 \mu \mathrm{M}$ dexamethasone (Sigma-Aldrich, St. Louis, MO, USA) dissolved in dimethyl sulfoxide (DMSO) and lithium chloride (LiCl) (Sigma-Aldrich, St. Louis, MO, USA) dissolved in distilled water was changed every 3 days throughout the experiments.

\section{shRNA interference}

Cell transfection was performed when the cells reached $80 \%$ confluence. The shRNA knockdown plasmids for DKK1 were purchased from GenePharma (GenePharma, Shanghai, China). Transfection was performed using the Lipofectamine 2000 reagent (Invitrogen, CA, USA) in Opti-MEM (Invitrogen, CA, USA) according to the manufacturer's protocol. TSCs were transfected for $4 \mathrm{~h}$, and the transfection mixture was replaced with culture medium.

$q P C R$

The mRNA expression levels of scleraxis, C/EBP $\alpha$, aP2, DKK1 were determined using qPCR. Total RNA was extracted from cells using TRIzol reagent, according to the protocol provided by the manufacturer (Takara, Dalian, China). cDNA was synthesized from total RNA using a Superscript III first-strand synthesis kit (TaKaRa). qPCR was performed using a SYBR Green RT-PCR kit (TaKaRa) and an ABI Prism 7900 Sequence Detection System (PE Applied Biosystems, Foster City, CA, USA). Expression levels were calculated relative to the expression of the housekeeping gene glyceraldehyde 3-phosphate dehydrogenase (GAPDH). The primer sequences used in this research are listed in Table 1.

\section{Protein extraction and Western blotting}

Cells were scraped and homogenized in lysis buffer (50 mM Tris-HCl, pH 8.0, 1 mM EDTA, 1\% Triton $\mathrm{X}-100,0.5 \%$ sodium deoxycholate, $0.1 \%$ sodium dodecyl sulfate (SDS), $150 \mathrm{mM} \mathrm{NaCl}$ ) containing a mixture of proteinase inhibitors (Thermo Fisher Scientific Inc., Rockford, IL, USA). Protein concentrations were measured using a BCA protein assay kit (Thermo Fisher Scientific Inc., Rockford, IL, USA). Protein samples (30 $\mu \mathrm{g} / \mathrm{lane})$ were resolved by SDS-polyacrylamide gel electrophoresis and transferred to polyvinylidene difluoride membranes. After blocking with $0.1 \%$ TBS-Tween containing $5 \%$ non-fat milk for $1 \mathrm{~h}$ at $20^{\circ} \mathrm{C}$, membranes were incubated sequentially with primary and secondary antibodies. The following primary antibodies were used: anti-aP2 (Abcam), C/EBP $\alpha$ (Abcam), DKK1 (Abcam), Phospho-GSK-3 $\alpha / \beta$ (ser21/9) (Cell Signaling, Danvers, MA, USA), Phospho-GSK-3 $\alpha / \beta$ (tyr279/tyr216) (Abcam), anti-active $\beta$-catenin (Millipore, Billerica, MA, USA). The results were visualized and images captured using a LiCor Odyssey Imager (LI-COR Biosciences, Lincoln, NE, USA).

Immunostaining

P3 TSCs were grown on glass coverslips, and cytospins or smear preparations were made. Cells were incubated with diluted anti-active $\beta$-catenin antibody (Millipore) in 5\% BSA in PBS-Tween-20 (PBST) overnight at $4{ }^{\circ} \mathrm{C}$. Then, the cells were incubated with Alexa Fluor 546 donkey anti-rabbit IgG (1:500) (Invitrogen, San Diego, CA, USA) in $5 \%$ BSA for $1 \mathrm{~h}$ at room temperature in the dark. The samples were also incubated with $0.5 \mu \mathrm{g} / \mathrm{ml}$ DAPI (Beyotime, Shanghai, China)
Table 1. qPCR primers

\begin{tabular}{llc}
\hline Gene & \multicolumn{1}{c}{ Primer sequences } & Product size (bp) \\
\hline ap2 & F: CGAGATTTCCTCAAACTGGG & 199 \\
& R: CTTGTAGAAGTCACGCCTTTC & \\
C/EBPo & F: GGTGGATAAGAACAGCAACG & 137 \\
& R: GGTCATTGTCACTGGTCAAC & \\
DKK1 & F: TTGCCGAAAGCGCAGGAAC & 108 \\
& R: CCTCGAGGTAAATGGCTGTG & \\
Scleraxis & F: GCAAGCTCTCCAAGATTGAG & 281 \\
& R: CGTCTTCTGTCACGGTCTT & \\
GAPDH & F: TGACTTCAACAGCAACTC & 110 \\
& R: TGTAGCCATATTCATTGTCA & \\
\hline
\end{tabular}




\section{Cellular Physiology Cell Physiol Biochem 2015;37:1738-1749 \begin{tabular}{l|l|}
\hline DOI: 10.1159/000438538 & O 2015 S. Karger AG, Basel \\
\hline
\end{tabular} www.karger.com/cpb \\ Chen et al.: Dickkopf1 Promotes rTSCs to Differentiate into Adipocytes}

for $2 \mathrm{~min}$. Coverslips were mounted using mounting medium, and the coverslips were sealed with nail polish to prevent drying and movement under the microscope. Five areas from two sections obtained from each patient were randomly selected and observed under $200 \times$ magnification using a Zeiss LSM-780 confocal microscopic system (Carl Zeiss, Gottingen, Germany).

\section{Oil red staining and HE staining}

Cultured TSCs were plated on cover slides in 24-well plates and treated with or without dexamethasone, DKK1 shRNA plasmid or LiCl in the culture medium for 21 days. Next, the cells were washed with phosphate-buffered saline (PBS), fixed with 4\% paraformaldehyde and stained with Oil Red O. Adipocytes were photographed under a microscope at $400 \times$ magnification. Human Achilles tendon specimens were obtained from patients in the Department of Orthopedic Surgery, Southwest Hospital, Third Military Medical University, Chongqing, China, with written consent. Experiments were approved by the institutional ethics committee. Human Achilles tendon specimens from cases of tendon rupture caused by trauma or dexamethasone treatment were fixed with $4 \%$ paraformaldehyde. Five sections were cut at a thickness of 4 $\mathrm{mm}$ and stained with hematoxylin and eosin.

\section{Statistical analysis}

Statistically significant differences were determined using Student's t-test or two-way analysis of variance. A P-value of $\leq 0.05$ was considered significant. All values are presented as the means \pm standard deviation (SD).

\section{Results}

Dex induces TSCs to differentiate into adipocytes

We studied the effects of different concentrations of Dex on TSC differentiation by examining the expression of tenogenic differentiation transcription factor scleraxis and the adipogenic differentiation transcription factor C/EBP $\alpha$ via $\mathrm{qPCR}$. Rat TSCs were treated by different concentration of Dex for 3 days. Dex at $1 \mathrm{nM}$ has no effect on the expression of either scleraxis or $\mathrm{C} / \mathrm{EBP} \alpha$, Dex at $10 \mathrm{nM}$ promoted scleraxis expression, and Dex at 100 $\mathrm{nM}$ of Dex had no effect on scleraxis expression, while Dex at the high concentration of 1 $\mu \mathrm{M}$ inhibited scleraxis expression. Dex at $10 \mathrm{nM}$ inhibited C/EBP $\alpha$ expression, and the high concentrations of Dex, $100 \mathrm{nM}$ and $1 \mu \mathrm{m}$, promoted C/EBP $\alpha$ expression (Fig. 1A-B).

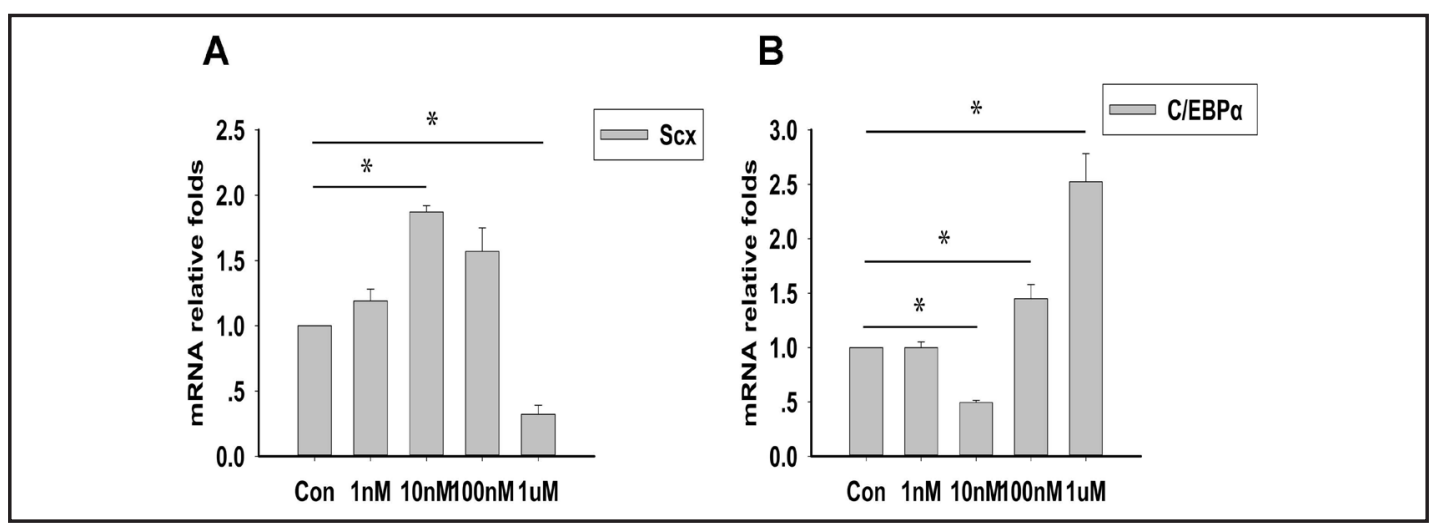

Fig. 1. Effects of different concentrations of dexamethasone on TSC differentiation. (A-B) qPCR analysis shows that $1 \mathrm{nM}$ Dex had no significant effect on the expression of scleraxis or C/EBP $\alpha$ in TSCs. (A) Dex at $10 \mathrm{nM}$ promoted mRNA expression of scleraxis in TSCs, $100 \mathrm{nM}$ of Dex had no significant effect on scleraxis expression in TSCs, and $1 \mu \mathrm{M}$ of Dex inhibited scleraxis expression. (B) Dex at $10 \mathrm{nM}$ inhibited C/EBP $\alpha$ expression, and Dex at $100 \mathrm{nM}$ or $1 \mu \mathrm{M}$ promoted C/EBP $\alpha$ expression. Relative mRNA expression levels were normalized to GAPDH. The data are presented as the means \pm SD of three independent experiments. $*: \mathrm{P}<0.05, \mathrm{~N}=3$. 


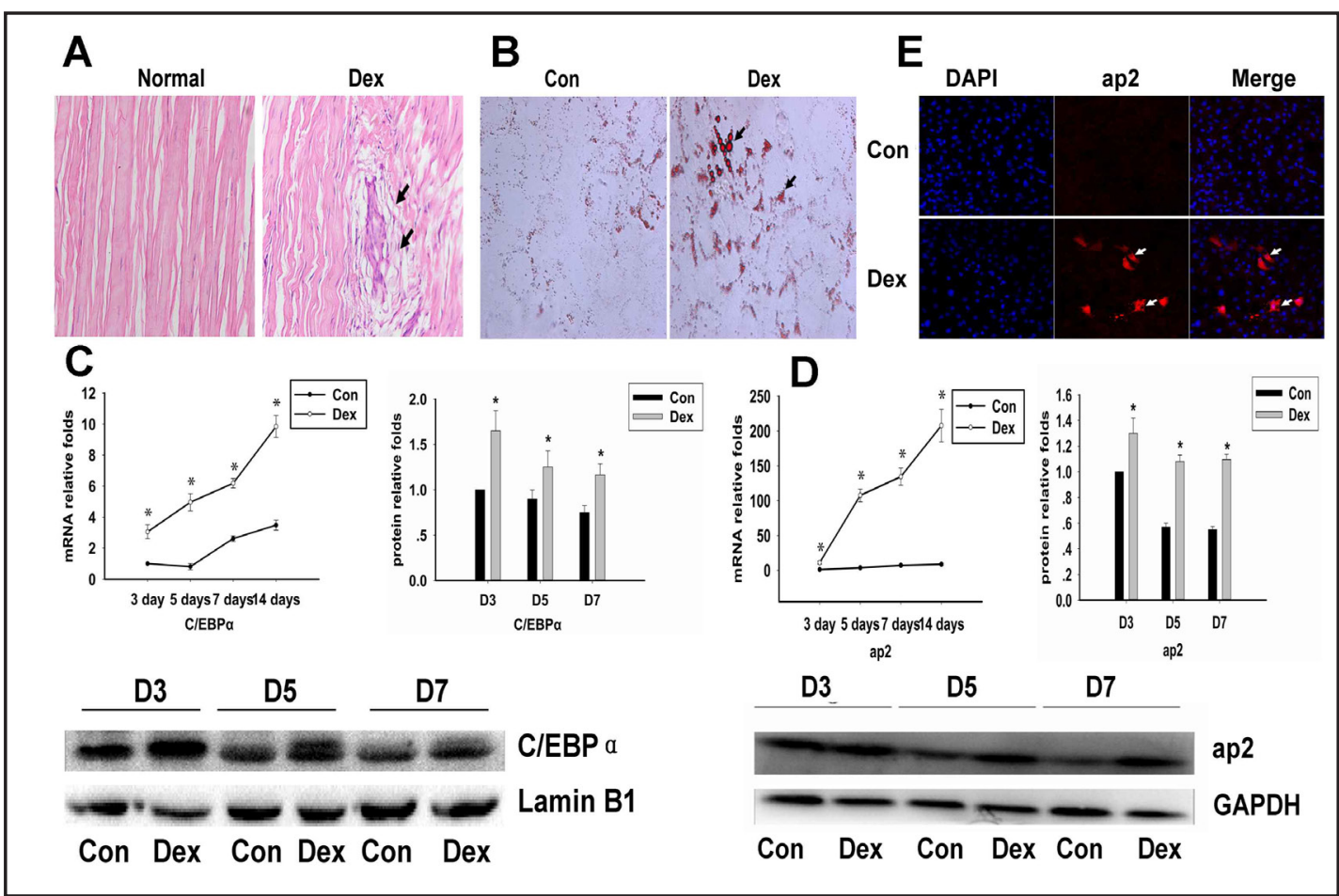

Fig. 2. Dex promoted TSCs to differentiate into adipocytes. (A) HE staining shows that Achilles tendon ruptures caused by trauma contained orderly collagen fibers, whereas the Achilles tendon ruptures caused by Dex contained irregularly arranged collagen fibers and adipose tissue metaplasia (black arrows indicate adipose tissue metaplasia, 200x). N = 3. (B) After TSCs were treated with Dex for 21 days, mature adipocyte formation was observed through oil red staining (black arrows indicate lipid droplet, $400 \times$ ). $N=3$. (C-D) After TSCs were treated with Dex for 3, 5, 7, and 14 days, C/EBP $\alpha$ and aP2 expression levels were increased at both mRNA and protein levels. qPCR showed that C/EBP $\alpha$ and aP2 expression levels increased with longer exposure to Dex. Relative mRNA and protein expression levels were normalized to GAPDH. The data are presented as the means \pm SD of three independent experiments. *: $\mathrm{P}<0.05, * *$ : $\mathrm{P}<0.01, \mathrm{~N}=3$. (E) After treating TSCs with Dex for 7 days, aP2-positive cells were generated, and some of those cells contained cytosolic lipid droplet-like structures (white arrows point to aP2-positive cells $200 \times$ ). N = 3 .

First, we used HE staining to compare the histological differences between the Achilles tendon ruptured by acute trauma and the Achilles tendon ruptured by long-term systemic administration of Dex. Collagen fibers in the Achilles tendon ruptures caused by trauma were arranged in an orderly manner, whereas the collagen fibers in the Achilles tendon ruptures caused by Dex were arranged irregularly and were accompanied by adipose tissue metaplasia (Fig. 2A). It is well known that the cytosol of mature adipocytes contains lipid droplets. After TSCs were treated with $1 \mu \mathrm{M}$ Dex for 21 days, the formation of lipid droplets in the cytosol was observed through oil red staining (Fig. 2B). After TSCs were treated with Dex for 3 days, 5 days, 7 days and 14 days, qPCR showed increases in the adipogenic differentiation markers aP2 and C/EBP $\alpha$. With time, aP2 and C/EBP $\alpha$ levels were gradually increased in both the control group and the experimental group (Fig. 2C, F). Western blotting results showed that after treating TSCs with Dex, aP2 and C/EBP $\alpha$ protein expression levels also increased (Fig. 2D-E, G-H). Immunofluorescence assays of aP2-positive cells showed that after treatment of TSCs with Dex for 7 days, large numbers of aP2-positive cells had formed, and the cytosol of some of the positive cells contained lipid droplet-like structures (Fig. 2I).

Dex inhibits the canonical WNT/ $\beta$-catenin pathway

Dickkopf (Dkk) family members specifically inhibit canonical WNT signaling by binding as high-affinity antagonists to LRP co-receptors [17]. We treated TSCs with $1 \mu \mathrm{M}$ Dex for 3, 


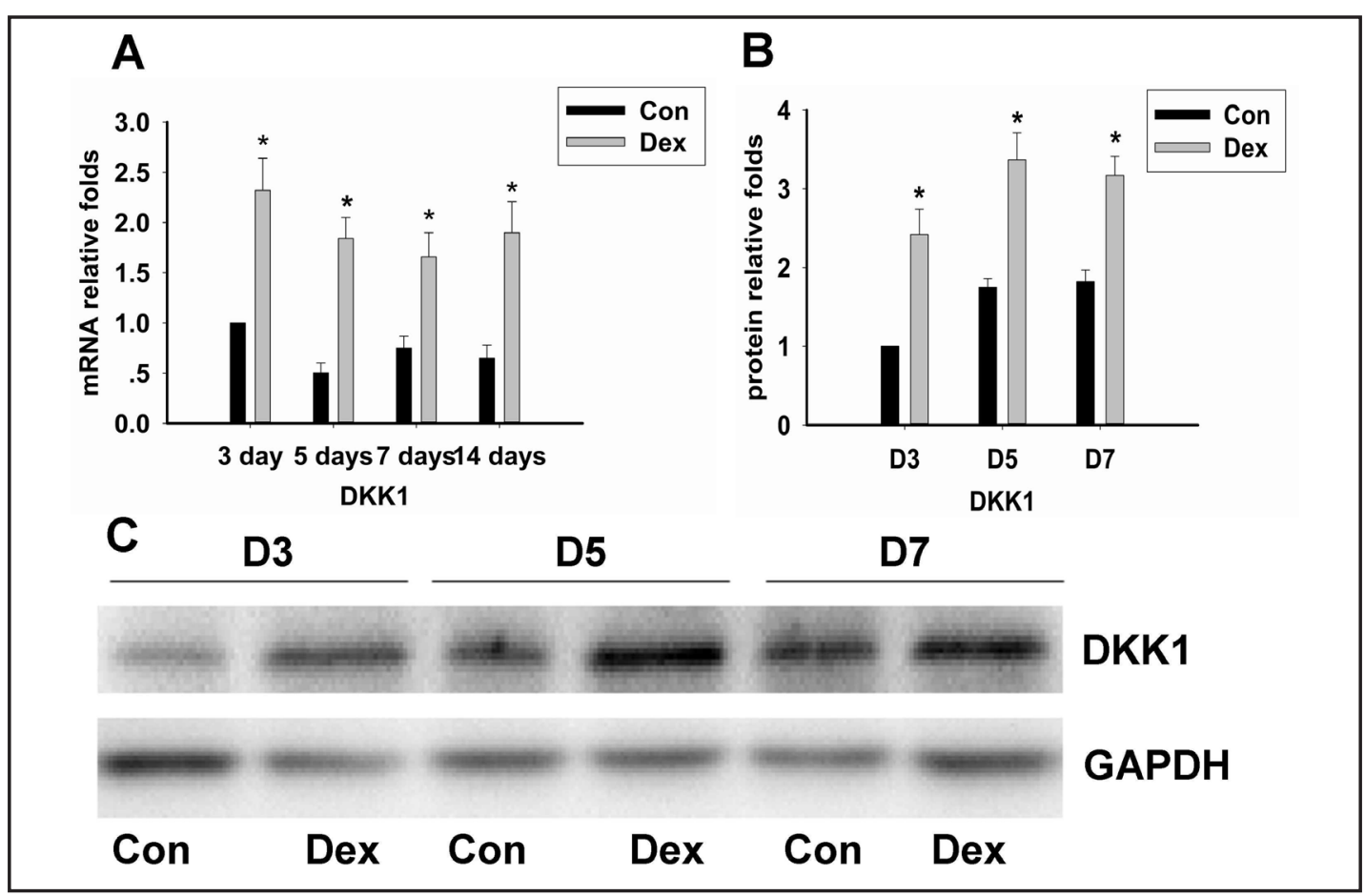

Fig. 3. Dex promoted DKK1 expression in rat TSCs. (A-C) When TSCs were treated with Dex for 3, 5, 7 or 14 days, both (A) mRNA and (B-C) protein expression levels of DKK1 were increased. Relative mRNA and protein expression levels were normalized to GAPDH. The data are presented as the means \pm SD of three independent experiments. $*$ : $\mathrm{P}<0.05, \mathrm{~N}=3$.

5, 7 and 14 days, and we found that Dex increased DKK1 mRNA and protein expression (Fig. $3 \mathrm{~A}-\mathrm{C}$ ). The canonical WNT signaling pathway converges on the transcriptional regulator $\beta$-catenin [18]. After GSK-3 $\beta$ is activated, it can form a degradation complex with $\beta$-catenin to promote $\beta$-catenin degradation. P-GSK-3 $\beta$ (ser9) is the inactive form of GSK-3 $\beta$, while P-GSK$3 \beta$ (tyr216) is the active form GSK-3 $\beta$ [19]. To study the effect of Dex on the canonical WNT pathway, TSCs were treated with $1 \mu \mathrm{M}$ Dex for 3, 5, or 7 days. Through Western blotting, we found that with 3 days of Dex treatment, both P-GSK-3 $\beta$ (ser9) and P-GSK-3 $\beta$ (tyr216) showed little change, whereas with Dex treatment for 5 days or 7 days, P-GSK-3 $\beta$ (ser9) decreased, and P-GSK-3 $\beta$ (tyr216) increased (Fig. 4A-D). When TSCs were treated with Dex for 3 days, $\beta$-catenin levels did not show a significant decrease, whereas with treatment for 5 days or 7 days, the expression of $\beta$-catenin decreased significantly (Fig. 4E-F). Immunohistochemistry showed that treatment of TSCs with Dex for 7 days significantly decreased the expression of $\beta$-catenin in the cytoplasm and nucleus (Fig. 4G-H).

DKK1 knockdown attenuated the inhibition of Dex on the canonical WNT pathway and induced adipogenic differentiation of TSCs

To investigate the role of DKK1 in the Dex-induced inhibition of the canonical WNT/ $\beta$ catenin pathway in TSCs and the Dex-induced promotion of adipogenic differentiation of TSCs, we used an shRNA plasmid to knock down DKK1, and we examined the changes to the canonical WNT/ $\beta$-catenin pathway in TSCs and the changes in adipogenic differentiation of TSCs after 5 days of interference. Western blotting showed that DKK1 interference attenuated the down regulation of Dex to P-GSK-3 $\beta$ (ser9) (Fig. 5A-B) and the up regulation of Dex to P-GSK-3 $\beta$ (tyr216) (Fig. 5C-D). Meanwhile, DKK1 knockdown up regulated active $\beta$-catenin and attenuated the Dex-induced down regulation of active $\beta$-catenin (Fig. 5E-F). qPCR and Western blotting showed that DKK1 knockdown attenuated the Dex-induced up regulation of aP2 and C/EBP $\alpha$ (Fig. 5G-J). Immunofluorescence showed that DKK1 interference reduced 


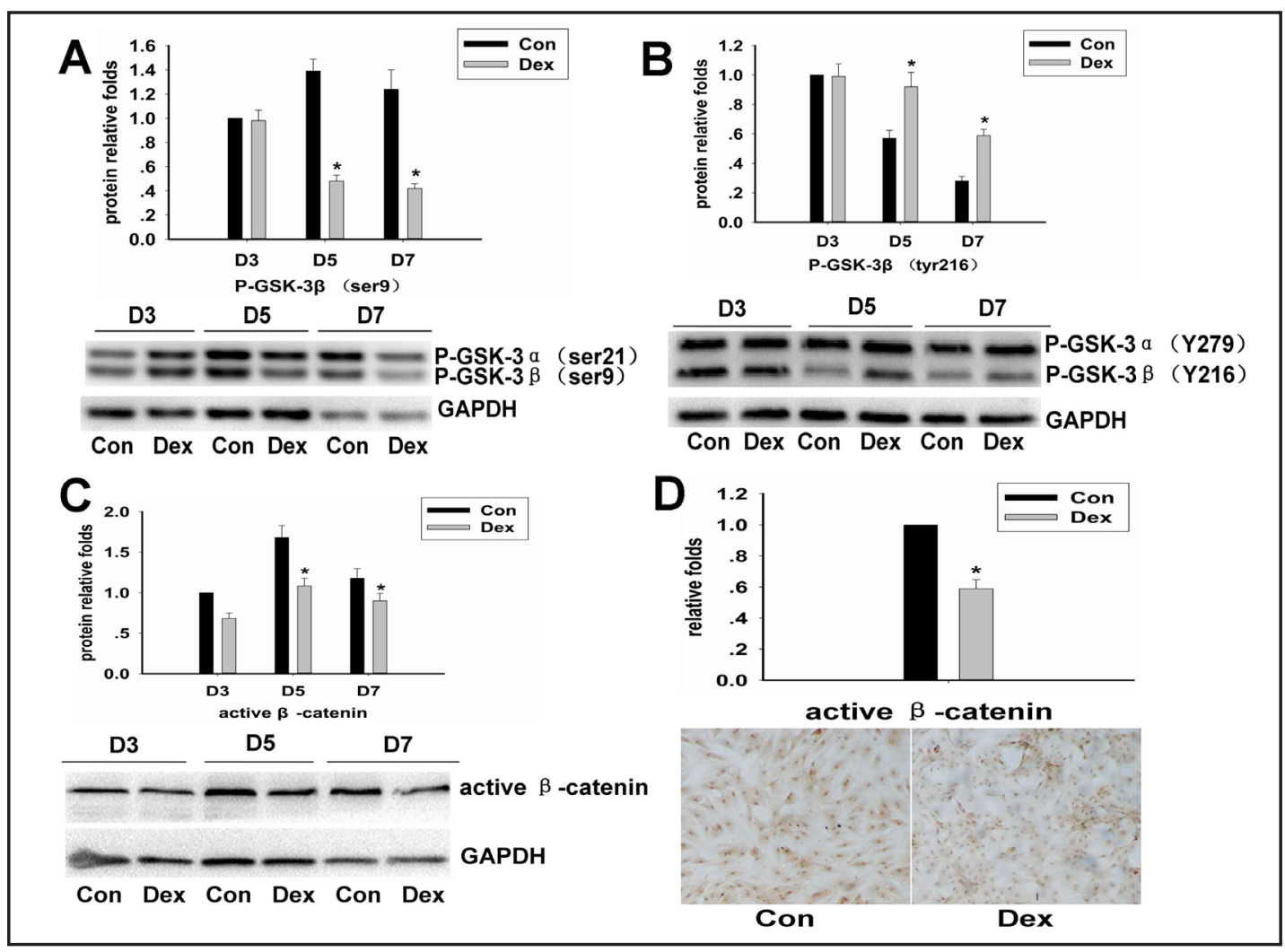

Fig. 4. Dex inhibited rat TSCs canonical WNT pathway. (A-B) Western blotting showed that when Dex acted on TSCs for 3 days, there was no significant change in both P-GSK-3 $\beta$ (ser9) and P-GSK-3 $\beta$ (tyr216) compared with the control group; when Dex acted for 5 days and 7 days, P -GSK-3 $\beta$ (ser9) decreased, while the P-GSK-3 $\beta$ (tyr216) increased. $N=3$. (C) Western blotting showed that after 3 days the decrease of $\beta$-catenin was not significant, while after 5 days and 7 days, $\beta$-catenin expression decreased. (D) Through immunocytochemistry staining, we found that when Dex acted for 7 days, $\beta$-catenin expression significantly decreased $(200 \times)$. $N=3$.

the formation of Dex-induced aP2-positive cells in TSCs (Fig. 5K). Oil red staining showed that DKK1 knockdown reduced the Dex-induced formation of mature adipocytes (Fig. 5L).

\section{LiCl attenuated the Dex-induced inhibition of the canonical WNT pathway in TSCs and the induction of adipogenic differentiation of TSCS}

By inhibiting GSK-3 $\beta$, LiCl reduced the binding of GSK-3 $\beta$ to $\beta$-catenin and the degradation of $\beta$-catenin, thereby activating the canonical WNT pathway. We examined the effects of Dex and LiCl on P-GSK-3 $\beta$ (ser9), P-GSK-3 $\beta$ (tyr216), and active $\beta$-catenin in TSCs. LiCl elevated P-GSK-3 $\beta$ (ser9) (Fig. 6A-B), lowered P-GSK-3 $\beta$ (tyr216) (Fig. 6C-D), and reduced the degradation of active $\beta$-catenin (Fig. $6 \mathrm{E}-\mathrm{F}$ ). LiCl attenuated the Dex-induced down regulation of P-GSK-3 $\beta$ (ser9), up-regulation of P-GSK-3 $\beta$ (tyr216) and down regulation of active $\beta$-catenin in TSCs (Fig. 6A-F). LiCl also antagonized the Dex-induced adipogenic differentiation of TSCs (Fig. 6G-L).

\section{Discussion}

In summary, the reasons for spontaneous tendon rupture and the difficulty of tendon self-repair after the use of Dex may be as follows: 1 . Dex inhibits tendon cell proliferation [20]; 2. Dex inhibits the migration of tendon cells [21]; 3. Dex inhibits the synthesis and 


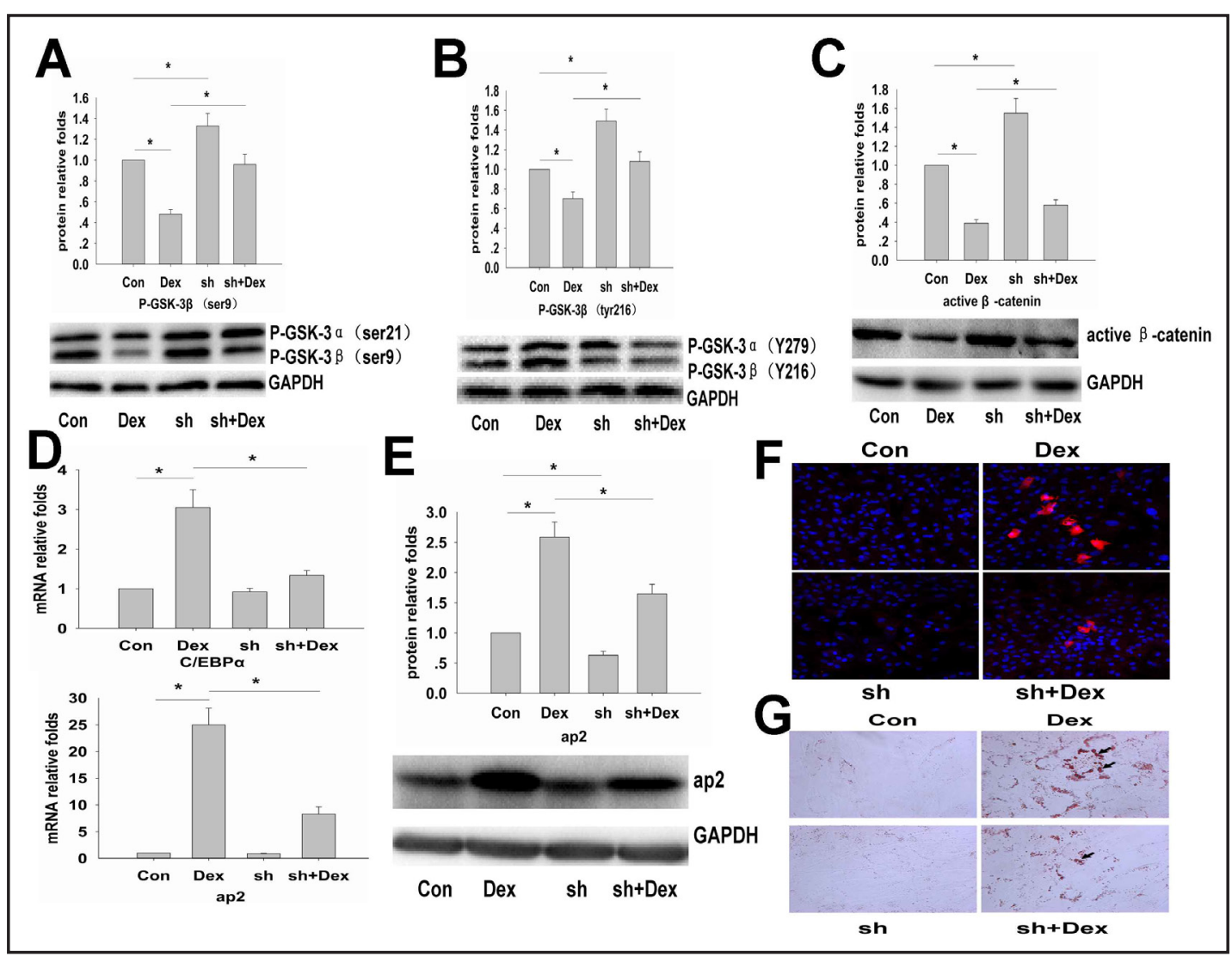

Fig. 5. shRNA targeting DKK1 attenuated the Dex-induced inhibition of the WNT signaling pathway in rat TSCs and the Dex-induced increase in adipogenic differentiation of TSCs. (A) Western blotting results showed that shRNA targeting DKK1 attenuated the Dex-induced down regulation of P-GSK-3 $\beta$ (ser9), N = 3. (B) DKK1 knockdown attenuated the Dex-induced up regulation of P-GSK-3 $\beta$ (tyr216), N = 3. (C) DKK1 knockdown attenuated the Dex-induced down regulation of active $\beta$-catenin, $N=3$. (D-E) qPCR and Western blotting results showed that DKK1 shRNA interference reduced the up regulation of Dex to aP2 and C/EBP $\alpha$, $\mathrm{N}=3$. Relative mRNA expression levels were normalized to GAPDH. The data are presented as the means \pm SD of three independent experiments. *: P < 0.05. (F) Immunofluorescence showed that DKK1 interference reduced the promotion of aP2-positive cell formation in TSCs by Dex, N = 3. (G) Oil red staining showed that DKK1 knockdown reduced the formation of mature adipocytes, $\mathrm{N}=3$.

secretion of type I collagen and other important tendon matrix proteins by tendon cells [2224]. Although TSCs account for only approximately $4-6 \%$ of cells in the tendon [11], their strong ability to proliferate and to differentiate into tendon cells makes them very important for both self-renewal and repair of tendon [11,25].

There are three possible sources of adipocytes formed after the injection of Dex: 1) Cells may be recruited from the vascular stroma of adipose tissue [14]. At present, there is no experimental evidence that other stem cells migrate to the tendon parenchyma to differentiate into adipocytes. 2) Mature tendon cells may be converted to adipocytes. Tempfer found mature adipocyte formation after treatment of human supraspinatus tendon-derived cells with crystalline glucocorticoid triamcinolone acetonide. That report suggested that a small group of cells from tendon-derived cell populations differentiated into adipocytes [26]. However, mature tendon cells do not have the capability to differentiate into adipocytes [27]. 3) Because TSCs can differentiate into adipocytes [8,27], the adipose metaplasia induced by Dex in tendon could come from the adipogenic differentiation of TSCs. Dex-induced adipogenic differentiation of TSCs may explain the Dex-induced spontaneous tendon rupture and the difficulty in tendon self-repair. Li found that Dex broke the balance 


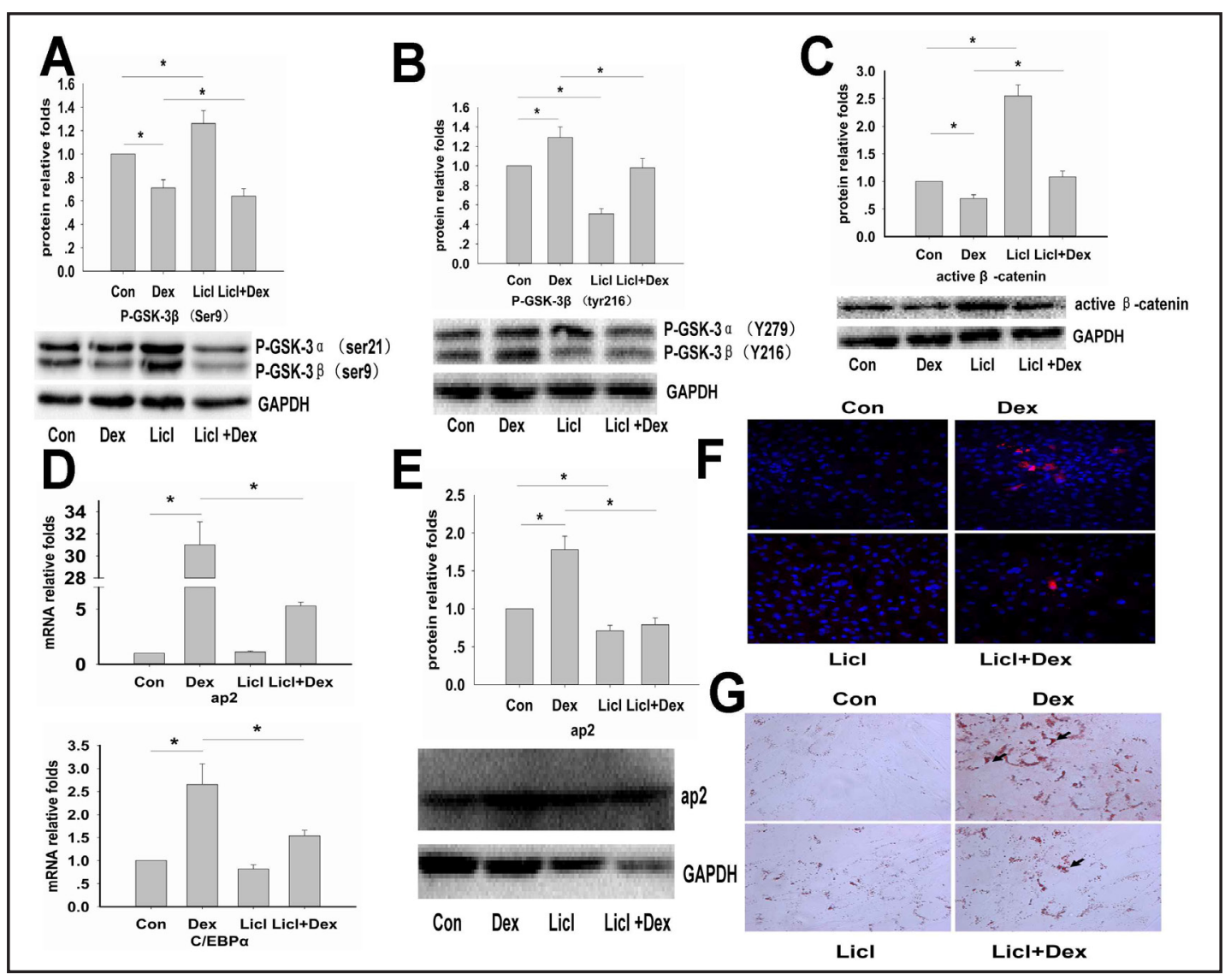

Fig. 6. In rat TSCs, LiCl attenuated the Dex-induced inhibition of WNT signaling and the Dex-induced adipogenic differentiation. (A) LiCl elevated P-GSK-3 $\beta$ (ser9). N = 3. (B) LiCl decreased P-GSK-3 $\beta$ (tyr216). $\mathrm{N}=3$. (C) LiCl reduced the degradation of active $\beta$-catenin. $\mathrm{N}=3$. (A-C) LiCl reduced Dex-induced changes in P-GSK-3 $\beta$ (ser9), P-GSK-3 $\beta$ (tyr216), and active $\beta$-catenin. N = 3. (D-G) LiCl also antagonized the Dexinduced adipogenic differentiation of TSCs. Relative mRNA expression levels were normalized to GAPDH. The data are presented as the means \pm SD of three independent experiments. *: $\mathrm{P}<0.05, \mathrm{~N}=3$.

between adipogenic and osteogenic differentiation of BMSCs, promoting the adipogenic differentiation of BMSCs, which plays an important role in the mechanism of Dex-induced osteoporosis [28].

Many factors have been found to induce preadipocytes to differentiate into adipocytes, including insulin-like growth factor 1 (IGF1), glucocorticoid, and cyclic AMP (cAMP). The tendon disease tissue itself may have adipose tissue metaplasia. To verify that the adipose tissue in ruptured tendon is caused by Dex, rather than being a pathological variation of tendon disease itself, we compared ruptured tendon caused by the long-term systemic administration of Dex due to autoimmune disease with ruptured tendon caused by acute trauma. We found that there was adipose tissue metaplasia in the ruptured tendon caused by systemic administration of Dex. In in vitro cell culture experiments, although the routine adipogenic differentiation induction solution DIM contains insulin and phosphodiesterase inhibitor methylisobutylxanthine (IBMX) in addition to Dex, Smas and Carlos found that the major mechanism by which Dex induces preadipocytes to differentiate into adipocytes is the inhibition of pref- 1 expression in preadipocytes $[29,30]$ and that Dex can independently inhibit pref-1 expression [30]; that is, Dex has the potential to independently induce stem cells to differentiate into adipocytes.

Sadowski estimated that the expression of more than 100 genes changes during the differentiation of preadipocytes into adipocytes. The most important genes include 
Fig. 7. Schematic illustration of the adipogenic differentiation induction of TSCs by Dex through DKK1/GSK$3 \beta / \beta$-catenin. Dex promoted the increase of DKK1 expression, causing downregulation of P-GSK-3 $\beta$ (ser9), up regulation of P-GSK-3 $\beta$ (tyr216), down regulation of $\beta$-catenin, and the promotion of adipogenic differentiation of TSCs.

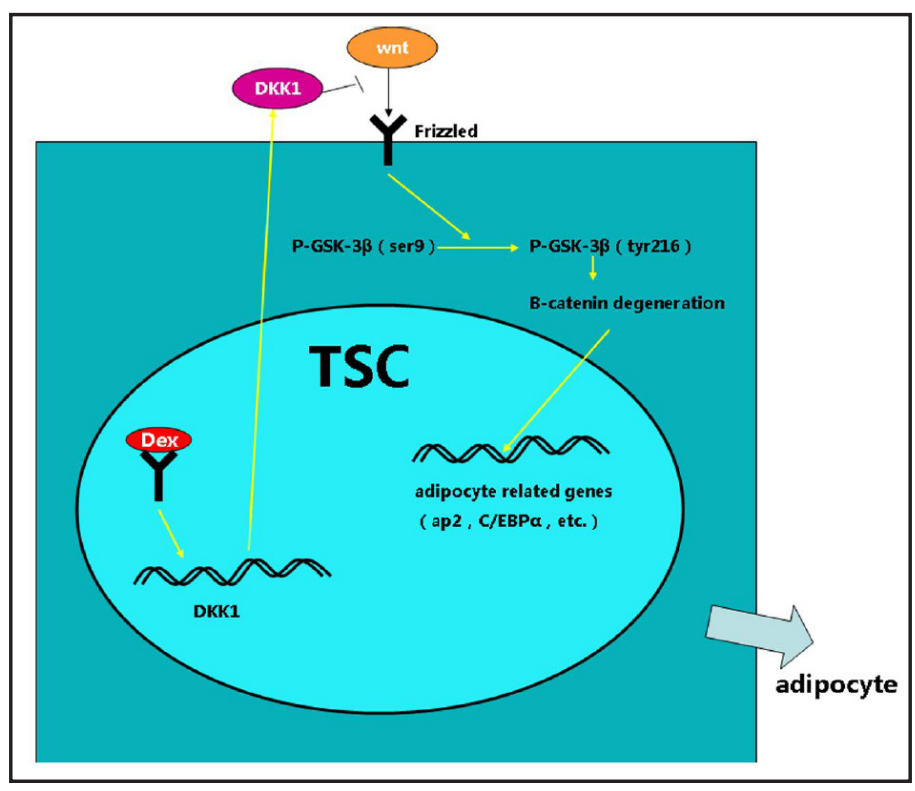

the C/EBP family, PPAR family, lipogenic enzymes, aP2, and various secreted factors. The expression of genes at different stage of differentiation from preadipocytes to adipocytes is different [31]. In the present study, we chose the early marker of adipogenic differentiation $\mathrm{C} / \mathrm{EBP} \alpha$ and late stage marker aP2. Dex significantly increased C/EBP $\alpha$ and aP2 expression at the transcriptional and translational levels. A mature adipocyte contains a single large fat droplet surrounded by a thin layer of cytoplasm that lies between the droplet and the plasma membrane [14]. Using oil red staining, we observed that Dex induced some TSCs to differentiate into mature adipocytes.

A study from Sona found that the activation of canonical WNT pathway can inhibit C/ EBP $\alpha$ and PPAR $\gamma$, thereby promoting the differentiation of stem cells into osteoblasts [32]. Wnts act at two points in the adipose development program, early in the program as activators of lineage commitment [33] and late in the program as inhibitors of adipocyte differentiation $[14,32,34]$. Through the inhibition of C/EBP $\alpha$ and PPAR $\gamma$, the WNT pathway maintains an undifferentiated condition to inhibit the differentiation of preadipocytes into adipocytes [34]. $\beta$-Catenin is the pivot molecule of the canonical WNT pathway, $\beta$-catenin-dependent signaling has been reported to promote both myogenesis [34] and osteogenesis [35] and to inhibit the differentiation of preadipocytes into adipocytes $[33,36]$. In addition, activation of the WNT signaling pathway enhances myogenesis [34] and osteogenesis [28, 37] and inhibits adipogenesis [28] in cultured MSCs. Masako reported that Dex could inhibit the canonical WNT signaling pathway through up regulation of the two inhibitory molecules DKK1 and Axin2 of the canonical WNT signaling pathway, while promoting the degradation of P-GSK$3 \beta$, thereby promoting the differentiation of preadipocytes into adipocytes $(32,33)$. Studies by Zhang found that Dex could promote TSCs to express PPAR $\gamma$. In vivo experiments further confirmed the formation of adipose tissue-like entity after the subcutaneous implantation of Dex-treated TSCs. Although PPAR $\gamma$ is frequently used as an adipogenic differentiation marker to examine adipogenic differentiation, our qPCR studies of Dex-treated TSCs showed no statistically significant difference in PPAR $\gamma$ mRNA expression compared with the control group. However, further study is needed to identify the reason. To clarify the mechanism by which Dex suppresses Wnt/ $\beta$-catenin signaling and promote adipogenesis, the present study examined the expression level of DKK1 in response to Dex stimulation and the effect of DKK1 expression on the adipogenesis of TSCs. Dex promotes the expression of the inhibitory molecule DKK1 of the canonical WNT pathway at the transcriptional and translational levels and down regulates P-GSK-3 $\beta$ (ser9), increases the expression of P-GSK-3 $\beta$ (tyr216), and promotes the degradation of active $\beta$-catenin, thereby inhibiting the canonical WNT pathway. Furthermore, DKK1 knockdown or the GSK-3 $\beta$ inhibitor LiCl can attenuate Dex- 


\begin{tabular}{|c|c|c|}
\hline Cellular Physiology & Cell Physiol Biochem 2015;37:1738-1749 & \\
\hline and Biochemistry & $\begin{array}{l}\text { Dol: 10.1159/000438538 } \\
\text { Published online: November 09, } 2015\end{array}$ & $\begin{array}{l}\text { O } 2015 \text { S. Karger AG, Basel } \\
\text { www.karger.com/cpb }\end{array}$ \\
\hline
\end{tabular}

induced adipogenesis in TSCs. Meanwhile, DKK1 knockdown can also attenuate the Dexinduced down regulation of P-GSK-3 $\beta$ (ser9) and up regulation of P-GSK-3 $\beta$ (tyr216).

Our results clearly demonstrate that Dex-induced adipogenesis is mediated by DKK1/ GSK-3 $\beta / \beta$-catenin (Fig. 7). DKK1 knockdown and GSK-3 $\beta$ inhibition may be useful targets for the treatment of GC-induced tendon rupture or weak self-repair ability.

\section{Limitations}

The present study found that Dex promoted adipogenic differentiation of TSCS and identified the underlying mechanism, but we did not compare whether TSCs or tendon cells are more important in tendon repair, nor did we identify the temporo-spatial changes in gene expression during the adipogenic differentiation of TSCs.

\section{Acknowledgments}

This work was supported by the National Natural Science Foundation of China (Grant No. 81230040). The authors report no potential conflicts of interest relevant to this article.

\section{Disclosure Statement}

None declared by any author.

\section{References}

1 Kleinman M, Gross AE: Achilles tendon rupture following steroid injection. Report of three cases. J Bone Joint Surg Am 1983;65:1345-1347.

2 McQuillan R, Gregan P: Tendon rupture as a complication of corticosteroid therapy. Palliative Medicine 2005;19:352-353.

3 Ford LT, DeBender J: Tendon rupture after local steroid injection. South Med J 1979;72:827-830.

4 Mikolyzk DK, Wei AS, Tonino P, Marra G, Williams DA, Himes RD, Wezeman FH, Callaci JJ: Effect of corticosteroids on the biomechanical strength of rat rotator cuff tendon. J Bone Joint Surg Am 2009;91:1172-1180.

5 Dean BJ, Lostis E, Oakley T, Rombach I, Morrey ME, Carr AJ: The risks and benefits of glucocorticoid treatment for tendinopathy: a systematic review of the effects of local glucocorticoid on tendon. Semin Arthritis Rheum 2014;43:570-576.

6 Barker N, Bartfeld S, Clevers H: Tissue-resident adult stem cell populations of rapidly self-renewing organs. Cell Stem Cell 2010;7:656-670.

7 Bi Y, He Y, Huang J, Su Y, Zhu GH, Wang Y, Qiao M, Zhang BQ, Zhang H, Wang Z, Liu W, Cui J, Kang Q, Zhang Z, Deng Y, Li R, Zhang Q, Yang K, Luu HH, Haydon RC, He TC, Tang N: Functional characteristics of reversibly immortalized hepatic progenitor cells derived from mouse embryonic liver. Cell Physiol Biochem 2014;34:1318-1338.

8 Bi YM, Ehirchiou D, Kilts TM, Inkson CA, Embree MC, Sonoyama W, Li L, Leet AI, Seo BM, Zhang L, Shi ST, Young MF: Identification of tendon stem/progenitor cells and the role of the extracellular matrix in their niche. Nature Medicine 2007;13:1219-1227.

9 Rui YF, Lui PP, Li G, Fu SC, Lee YW, Chan KM: Isolation and characterization of multipotent rat tendonderived stem cells. Tissue Eng Part A 2010;16:1549-1558.

10 Magnusson SP, Langberg H, Kjaer M: The pathogenesis of tendinopathy: balancing the response to loading. Nat Rev Rheumatol 2010;6:262-268.

11 Lui PP, Chan KM: Tendon-derived stem cells (TDSCs): from basic science to potential roles in tendon pathology and tissue engineering applications. Stem Cell Rev 2011;7:883-897.

12 Chen L, Liu JP, Tang KL, Wang Q, Wang GD, Cai XH, Liu XM: Tendon derived stem cells promote platelet-rich plasma healing in collagenase-induced rat achilles tendinopathy. Cell Physiol Biochem 2014;34:2153-2168.

13 Zhang JY, Keenan C, Wang JHC: The effects of dexamethasone on human patellar tendon stem cells: Implications for dexamethasone treatment of tendon injury. J OrthopRes 2013;31:105-110. 


\section{Cellular Physiology Cell Physiol Biochem 2015;37:1738-1749 \begin{tabular}{l|l} 
DOI: 10.1159/000438538 & (C) 2015 S. Karger AG, Basel
\end{tabular} www.karger.com/cpb \\ Chen et al.: Dickkopf1 Promotes rTSCs to Differentiate into Adipocytes}

14 Tang QQ, Lane MD: Adipogenesis: from stem cell to adipocyte. Annu Rev Biochem 2012;81:715-736.

15 Guo W, Flanagan J, Jasuja R, Kirkland J, Jiang L, Bhasin S: The effects of myostatin on adipogenic differentiation of human bone marrow-derived mesenchymal stem cells are mediated through crosscommunication between Smad3 and Wnt/beta-catenin signaling pathways. J Biolog Chem 2008;283:91369145.

16 Liu J, Chen L, Tao X, Tang K: Phosphoinositide 3-kinase/Akt signaling is essential for prostaglandin E2induced osteogenic differentiation of rat tendon stem cells. Biochem Biophys Res Commun 2013;435:514519.

17 Zorn AM: Wnt signalling: antagonistic Dickkopfs. Curr Biol 2001;11:R592-595.

18 D'Alimonte I, Lannutti A, Pipino C, Di Tomo P, Pierdomenico L, Cianci E, Antonucci I, Marchisio M, Romano M, Stuppia L, Caciagli F, Pandolfi A, Ciccarelli R: Wnt signaling behaves as a "master regulator" in the osteogenic and adipogenic commitment of human amniotic fluid mesenchymal stem cells. Stem Cell Rev 2013;9:642-654.

19 Gao Y, Liu Z, Zhang X, He J, Pan Y, Hao F, Xie L, Li Q, Qiu X, Wang E: Inhibition of cytoplasmic GSK-3beta increases cisplatin resistance through activation of Wnt/beta-catenin signaling in A549/DDP cells. Cancer Lett 2013;336:231-239.

20 Wong MW, Tang YN, Fu SC, Lee KM, Chan KM: Triamcinolone suppresses human tenocyte cellular activity and collagen synthesis. Clin Orthop Relat Res 2004;277-281.

21 Tsai WC, Tang FT, Wong MK, Pang JH: Inhibition of tendon cell migration by dexamethasone is correlated with reduced alpha-smooth muscle actin gene expression: a potential mechanism of delayed tendon healing. J Orthop Res 2003;21:265-271.

22 Poulsen RC, Carr AJ, Hulley PA: Protection against glucocorticoid-induced damage in human tenocytes by modulation of ERK, Akt, and forkhead signaling. Endocrinology 2011;152:503-514.

23 Wong MW, Tang YY, Lee SK, Fu BS: Glucocorticoids suppress proteoglycan production by human tenocytes. Acta Orthop 2005;76:927-931.

24 Wong MW, Lui WT, Fu SC, Lee KM: The effect of glucocorticoids on tendon cell viability in human tendon explants. Acta Orthop 2009;80:363-367.

25 Zhang J, Keenan C, Wang JH: The effects of dexamethasone on human patellar tendon stem cells: implications for dexamethasone treatment of tendon injury. J Orthop Res 2013;31:105-110.

26 Tempfer H, Gehwolf R, Lehner C, Wagner A, Mtsariashvili M, Bauer HC, Resch H, Tauber M: Effects of crystalline glucocorticoid triamcinolone acetonide on cultered human supraspinatus tendon cells. Acta Orthop 2009;80:357-362.

27 Zhang J, Wang JH: Characterization of differential properties of rabbit tendon stem cells and tenocytes. BMC Musculoskelet Disord 2010;11:10.

28 Li J, Zhang N, Huang X, Xu J, Fernandes JC, Dai K, Zhang X: Dexamethasone shifts bone marrow stromal cells from osteoblasts to adipocytes by C/EBPalpha promoter methylation. Cell Death Dis 2013;4:e832.

29 Pantoja C, Huff JT, Yamamoto KR: Glucocorticoid signaling defines a novel commitment state during adipogenesis in vitro. Mol Biol Cell 2008;19:4032-4041.

30 Smas CM, Chen L, Zhao L, Latasa MJ, Sul HS: Transcriptional repression of pref-1 by glucocorticoids promotes 3T3-L1 adipocyte differentiation. J Biol Chem 1999;274:12632-12641.

31 Gregoire FM, Smas CM, Sul HS: Understanding adipocyte differentiation. Physiol Rev 1998;78:783-809.

32 Kang S, Bennett CN, Gerin I, Rapp LA, Hankenson KD, Macdougald OA: Wnt signaling stimulates osteoblastogenesis of mesenchymal precursors by suppressing CCAAT/enhancer-binding protein alpha and peroxisome proliferator-activated receptor gamma. J Biol Chem 2007;282:14515-14524.

33 Bowers RR, Lane MD: Wnt signaling and adipocyte lineage commitment. Cell Cycle 2008;7:1191-1196.

34 Ross SE, Hemati N, Longo KA, Bennett CN, Lucas PC, Erickson RL, MacDougald OA: Inhibition of adipogenesis by Wnt signaling. Science 2000;289:950-953.

35 Bennett CN, Longo KA, Wright WS, Suva LJ, Lane TF, Hankenson KD, MacDougald OA: Regulation of osteoblastogenesis and bone mass by Wnt10b. Proc Natl Acad Sci U S A 2005;102:3324-3329.

36 Kennell JA, O'Leary EE, Gummow BM, Hammer GD, MacDougald OA: T-cell factor 4N (TCF-4N), a novel isoform of mouse TCF-4, synergizes with beta-catenin to coactivate C/EBPalpha and steroidogenic factor 1 transcription factors. Mol Cell Biol 2003;23:5366-5375.

37 Kohn AD, Moon RT: Wnt and calcium signaling: beta-catenin-independent pathways. Cell Calcium 2005;38:439-446. 